\title{
Supper lattice structure transformation based on nonstoichiometric bismuth oxychloride
}

\author{
Sujuan $\mathrm{Wu}^{1,2}$, Jianguo Sun ${ }^{1}$ and Shize $\mathrm{Yang}^{2}$ \\ ${ }^{1 .}$ Electron Microscopy Center of Chongqing University, College of Materials Science and Engineering, \\ Chongqing University, Chongqing 400044, China. \\ 2. Materials Science and Technology Division, Oak Ridge National Laboratory, Oak Ridge, Tennessee \\ 37831, USA.
}

Two-dimensional layered materials (2DLMs) with intriguing properties are hot topics of current interest, which have been extensively explored in photocatalyst, photocathodes, sensors and nanoelectronic devices et al [1-2]. They are usually featured by a single atom- thick or polyhedral-thick atomic layers with covalent or ionic bonding within each layer and van der Waals bonding. As such, the weak van der Waals interaction enables the possibilities of integrating 2DLMs with various nanoscale materials to create diverse van der Waals heterostructures (vdWHs) with functions that were not previously possible or tailoring their electronic and optoelectronic properties for functional devices [3].

The bismuth oxychloride $(\mathrm{BiOCl})$ is a typical 2DLM featured with layered structure of [Cl-Bi-O-Bi-Cl] slices stacking along the [001] direction by the van der Waals interaction through chlorine atoms [4]. To widen the application and tailor the conductor properties of 2DLM BiOCl, phases and polymorphs which can complement or enhance properties of this unique 2D ternary semiconducting system are explored in the $\mathrm{Bi}-\mathrm{O}-\mathrm{Cl}$ system, such as $\mathrm{Bi}_{3} \mathrm{O}_{4} \mathrm{Cl}, \mathrm{Bi}_{12} \mathrm{O}_{15} \mathrm{C}_{16}, \mathrm{Bi}_{24} \mathrm{O}_{31} \mathrm{Cl}_{10}[5,6]$. The previous studies show that $\mathrm{Bi}_{3} \mathrm{O}_{4} \mathrm{Cl}$ could be excited by the visible light and the photocurrent density is $15 \mu \mathrm{A} / \mathrm{cm}^{2}$, while the $\mathrm{Bi}_{24} \mathrm{O}_{31} \mathrm{Cl}_{10}$ with narrowed band gap of $2.8 \mathrm{eV}$ and the power conversion efficiency could be $1.5 \%$. Myung et al. reported that the intrinsic conductivity switches from p-type to n-type as the BiOCl transferred to an oxygen-rich $\mathrm{Bi}_{12} \mathrm{O}_{15} \mathrm{C}_{16}$ phase, and the photoconductivity increase of 80 times [7]. More interesting, a periodic super lattice structure appeared resulting from the atomic rearrangement of the $\mathrm{Bi}^{3+}, \mathrm{O}^{2-}$ and $\mathrm{Cl}^{-}$ ions. It is thereby expected that the super lattice periodicity and the resulting properties can be tailored by control of the stoichiometry in the $\mathrm{BiOCl}$ system.

In the present study, the in-situ fabrication of $\mathrm{Bi}_{12} \mathrm{O}_{15} \mathrm{C}_{16}$ and $\mathrm{Bi}_{24} \mathrm{O}_{31} \mathrm{Cl}_{10}$ super lattice structure are achieved by in-situ annealing $\mathrm{BiOCl}$. As shown in Figure1, the lattice spacing is determined to be 0.354 $\mathrm{nm}$, which corresponds to the (206) crystal plane in $\mathrm{Bi}_{24} \mathrm{O}_{31} \mathrm{Cl}_{10}$, as highlighted in Figure 1a. Due to the different ratio of $\mathrm{Bi} / \mathrm{O} / \mathrm{Cl}$ atoms, the Fast Fourier Transform (FFT) patterns of $\mathrm{A}, \mathrm{B}, \mathrm{C}$ (marked in Figure.1a) show an evolution process of super lattice structure with a tunable period. Different long period structures are significantly observed both in the FFT patterns (Figure. $1 \mathrm{e}-\mathrm{g}$ ) and the corresponding images (Figure. 1 b-d).

\section{References:}

[1] A. K. Geim, I. V. Grigorieva, Nature 499 (2013) 419-425.

[2] Z. Sun, H. Chang, ACS Nano 8 (2014) 4133-4156.

[3] Y. Liu, N. O. Weiss, X. Duan et al. Nature Reviews Materials 1 (2016)16042.

[4] J. Jiang, K. Zhao, X. Xiao, L. Zhang, J. Am. Chem. Soc. 134 (2012) 4473-4479.

[5] L. Wang, J. Shang, W. Hao, et al. Scientific reports 4 (2014) 7384. 
[6] C. Y. Wang, X. Zhang, X. N. Song, et al. ACS Appl. Mater. Interfaces 8 (2016) 5320-5326.

[7] Y. Myung, F. Wu, S. Banerjee et al. Chem. Mater. 27 (2015) 7710-7718.

The authors acknowledge funding from the National Natural Science Foundation of China (No.51302329) and the Fundamental Research Funds for the Central Universities (No.106112015CDJXY130010).
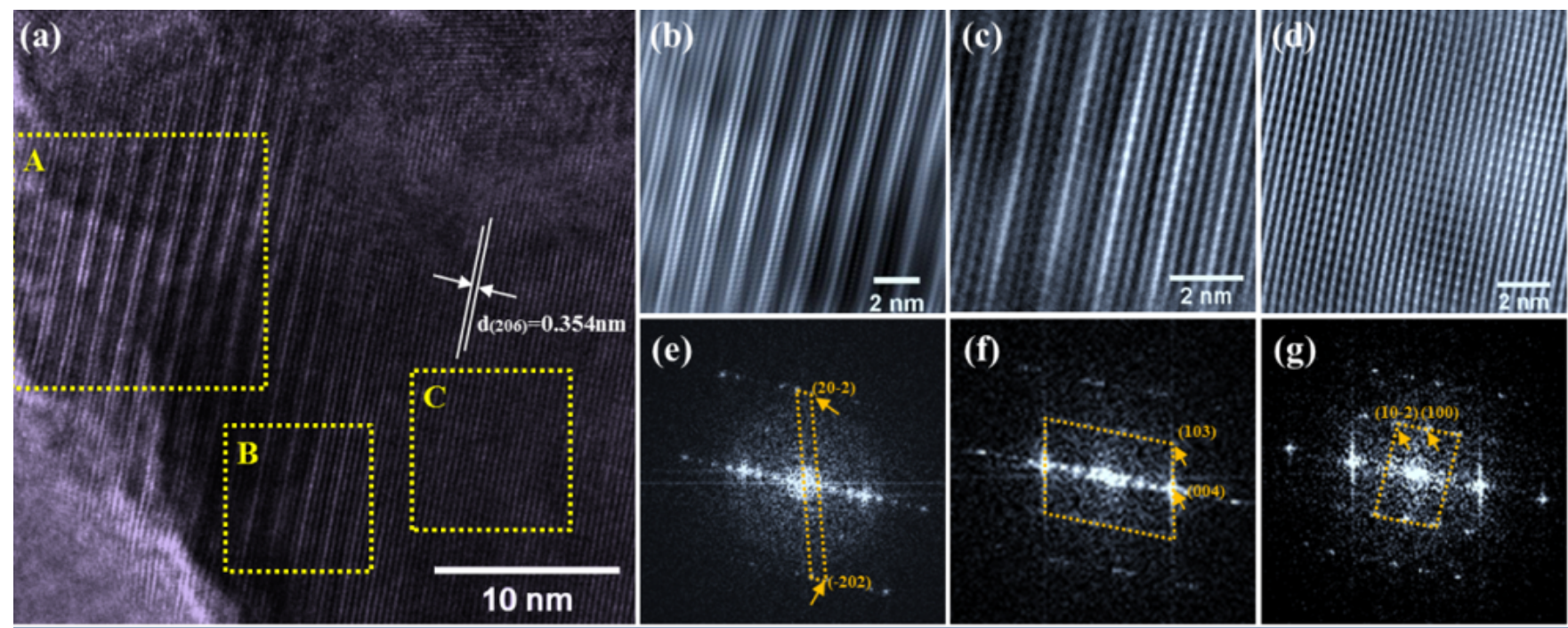

Figure 1. TEM images of $\mathrm{Bi}_{24} \mathrm{O}_{31} \mathrm{Cl}_{10}$ super lattice structure (a). Reverse FFT images (b-d) and FFT images (e-g) of the corresponding area of A, B, C marked in (a). 